\title{
Nutrición de Precisión para Dislipidemias en la Infección por el VIH/Sida
}

\author{
Precision Nutrition for Dyslipidemias in HIV/AIDS
}

\author{
Liliana Arias Castelán ${ }^{a}$, Marcos Galván $^{b}$, Guadalupe López-Rodríguez ${ }^{c}$
}

\begin{abstract}
:
The dyslipidemia associated with HIV/AIDS is multifactorial and constitutes a risk factor for the development of macrovascular complications: myocardial infarction and cerebrovascular disease, which affect the quality of life. At the beginning of the pharmacological treatments of patients with HIV/AIDS, dyslipidemias were associated with protease inhibitors (PI) and, later, with nucleoside reverse transcriptase inhibitors (NRTIs) and non-nucleoside reverse transcriptase inhibitors (NNRTIs). Evidence indicates that the use of these antiretroviral drugs increases the expression of the sterile regulatory element binding protein (SREBP) gene, which is regulated by the SREBP1 gene; thus, increasing the expression of lipogenic genes.

Precision nutrition is an approach that aims at the individualized nutrition of the patient where the phenotype, genotype and physical activity practices of the subject under evaluation are considered; the objective of this clinical case report is to characterize and describe a patient's dyslipidemia with HIV a precision nutrition approach based on the patient's particular conditions and the functioning of the SRBEP1 protein, which regulates the expression of genes involved in lipogenesis.
\end{abstract}

Keywords:

HIV/AIDS, Precision nutrition, Dyslipidemia, SREBP1, Antiretrovirals

\section{Resumen:}

La dislipidemia asociada a VIH/Sida es multifactorial y constituye un factor de riesgo para el desarrollo de complicaciones macrovasculares: infarto de miocardio y enfermedad cerebrovascular, las cuales afectan la calidad de vida. Al inicio de los tratamientos farmacológicos de pacientes con VIH/Sida, las dislipidemias se asociaron con los inhibidores de proteasas (IP), y más tarde, con los inhibidores de la transcriptasa reversa de nucleósidos (ITRNs) y los inhibidores de la transcriptasa reversa no nucleósidos (ITRNNs). La evidencia indica que el uso de estos fármacos antirretrovirales incrementa la expresión del gen sterol regulatory element binding proteins (SREBP), el cual es regulado por el gen SREBP1; incrementándose así la expresión de genes lipogénicos.

La nutrición de precisión es un abordaje que tiene como fin la nutrición individualizada del paciente, donde se consideran el fenotipo, genotipo y las prácticas de actividad física del sujeto a evaluar; el objetivo de este informe de caso clínico es caracterizar y describir las dislipidemias de un paciente con $\mathrm{VIH}$, un abordaje de nutrición de precisión basado en las condiciones particulares del paciente y el funcionamiento de la proteína SRBEP1, la cual regula la expresión de genes involucrados en la lipogénesis.

\section{Palabras Clave:}

VIH/sida, Nutrición de precisión, Dislipidemia, SREBP1, Antirretrovirales.

\section{Introducción}

El virus de inmunodeficiencia humana $(\mathrm{VIH})$ infecta a las células del sistema inmunitario, alterando o anulando su función. La infección produce un deterioro progresivo del sistema inmunitario, con la consiguiente inmunodeficiencia [1] y otras comorbilidades como son las enfermedades cardiovasculares.

Actualmente, las complicaciones cardiovasculares representan la primera causa de muerte a nivel global con 17.3 millones al año [2]. La terapia antirretroviral

\footnotetext{
"Centro Ambulatorio de Atención y Prevención de SIDA e Infecciones de Transmisión Sexual, Servicios de Salud de Hidalgo. Universidad Autónoma del Estado de Hidalgo, Instituto de Ciencias de la Salud, Maestría en Nutrición Clínica. Email: liliana_arias6@hotmail.com.

${ }^{\mathrm{b}}$ Universidad Autónoma del Estado de Hidalgo, Instituto de Ciencias de la Salud, Área Académica de Nutrición, https://orcid.org/00000002-3254-4470, Email: mgalvan@uaeh.edu.mx.

${ }^{c}$ Autor de correspondencia, Universidad Autónoma del Estado de Hidalgo, Instituto de Ciencias de la Salud, Área Académica de Nutrición, https://orcid.org/0000-0001-5432-0382, Email: glopez@uaeh.edu.mx.
} 
altamente activa (TARAA) en pacientes con infección por $\mathrm{VIH}$ disminuyó la morbimortalidad del padecimiento. Sin embargo, la dislipidemia y otras alteraciones metabólicas son parte de las complicaciones más frecuentes en los pacientes infectados por el VIH que reciben tratamiento antirretroviral [3]. Los síntomas son similares a lo observado en el síndrome metabólico [4].

La infección por VIH asintomática se asocia con una disminución temprana en las concentraciones plasmáticas de colesterol de alta densidad (HDL) [5]. La progresión hacia enfermedad sintomática y a síndrome de inmunodeficiencia adquirida (Sida) causa mayor disminución del colesterol HDL, aumento del colesterol total, elevación de triglicéridos y acumulación de subclases pequeñas y densas de las lipoproteínas de baja densidad (LDL). Otra característica de los pacientes con $\mathrm{VIH} / \mathrm{SIDA}$ es que los niveles de colesterol HDL son menores en aquellos con cuentas bajas de linfocitos $\mathrm{CD} 4^{+}[6]$.

Las dislipidemias también se han asociado el uso de fármacos antirretrovirales, explicadas por el incremento en el nivel de activación de sterol regulatory element binding proteins (SREBP), el cual es regulado por el gen SREBP1 [7]. En animales tratados con Ritonavir® se observa una elevación de SREBP-1 y 2, proteínas que modifican la expresión de los genes reguladores del metabolismo lipídico, lo que induciría a un aumento de colesterol y triglicéridos. La acumulación de SREBPs activados en el núcleo de las células, sugiere que los genes que responden a estos factores de transcripción pueden inducirse constitutivamente después del tratamiento con Ritonavir [8].

La regulación de la expresión de genes es uno de los objetivos de la nutrición de precisión [9], utilizando nutrimentos 0 compuestos bioactivos se pueden modificar la cantidad de transcrito de un gen y por lo tanto la actividad de las proteínas (nutrigenómica). La primera evidencia de la regulación transcripcional de SREBP1c se obtuvo en ensayos de ayuno/ingesta en roedores, demostrando que estos cambios nutricionales regulan la expresión de SREBP1c en hígado [10], tejido adiposo [11] y músculo, mientras que provocan sólo ligeros efectos en SREBP1a y SREBP2. Así, la expresión de SREBP1c disminuye durante el ayuno, pero aumenta marcadamente cuando los animales son realimentados con una dieta rica en carbohidratos.

\section{Caso Clínico}

Paciente masculino de 43 años de edad, el cual cursa con diagnóstico de VIH/Sida C3 desde 2010. Actualmente se encuentra bajo tratamiento antirretroviral con base en Lopinavir $200 \mathrm{mg} /$ Ritonavir $50 \mathrm{mg}$ y Zidovudina $300 \mathrm{mg} / \mathrm{Lamivudina} 150 \mathrm{mg}$. Su carga viral es menos de 40 copias y CD4 $4^{+}$de 511 células.

Antecedentes heredo familiares: diabetes mellitus tipo 2 e hipertensión arterial sistémica (rama materna), resto interrogado y negado. Antecedentes personales patológicos: tuberculosis pulmonar hace 9 años, resto interrogado y negado. Antecedentes personales no patológicos: alcoholismo social ocasional, niega otras toxicomanías.

Paciente con antecedente de dislipidemia mixta severa, registrada poco después del inicio de tratamiento antirretroviral con niveles de triglicéridos de $772 \mathrm{mg} / \mathrm{dL}$ como máximo y $308 \mathrm{mg} / \mathrm{dL}$ como mínimo; y con niveles de colesterol total de $405 \mathrm{mg} / \mathrm{dL}$ como máximo y 273 $\mathrm{mg} / \mathrm{dL}$ como mínimo. El resto de los bioquímicos dentro de límites normales.

Actualmente asintomático, en alerta, hidratado, adecuada coloración de tegumentos y mucosas, campos pulmonares normales, abdomen plano, blando, depresible, no doloroso. Con peristalsis normal.

Al momento de la consulta nutricional refiere tener un trabajo estable, es pastelero de oficio, tiene un nivel socioeconómico medio; sin embargo, en su empleo no saben de su diagnóstico, lo que le dificulta cuidar de su alimentación; ya que no quiere que sospechen que está enfermo. Tiene una complexión corporal delgada. Refiere hacer 20 minutos de ejercicio aeróbico (salta la cuerda) dos veces por semana desde hace 3 meses.

Tabla 1. Datos antropométricos del paciente

\begin{tabular}{|c|c|c|c|c|}
\hline $\begin{array}{c}\text { Talla } \\
(\mathbf{m})\end{array}$ & $\begin{array}{c}\text { Peso } \\
\text { actual } \\
(\mathrm{Kg})\end{array}$ & $\begin{array}{c}\text { Peso } \\
\text { habitual } \\
(\mathrm{Kg})\end{array}$ & $\begin{array}{c}\mathrm{IMC} \\
\left(\mathrm{Kg} / \mathbf{m}^{2}\right)\end{array}$ & $\begin{array}{c}\text { Estado } \\
\text { Nutricional }\end{array}$ \\
\hline 1.64 & 64 & 65 & 23.8 & $\begin{array}{c}\text { Peso } \\
\text { Normal }\end{array}$ \\
\hline
\end{tabular}

De acuerdo al análisis de la dieta evaluada con un recordatorio de 24 horas, el paciente registró un consumo de energía de $2257.2 \mathrm{Kcal}, 17.5 \%$ mayor (1920 $\mathrm{Kcal} /$ ) a la ingesta diaria recomendada (IDR), con una distribución porcentual entre carbohidratos/lípidos/proteínas de 48.0/32.7/18.3, respectivamente. Se identificó una ingesta recomendada de nutrientes (RDA) mayor de ácidos grasos 
monoinsaturados (10.3\% vs RDA $9 \%$ ) y saturados (13.8\% vs RDA $\leq 10 \%)$ y menor de poliinsaturados $(5.9 \%$ vs RDA 6-11\%) [12], así como un elevado consumo de colesterol (539.3 mg).

La ingesta de algunos micronutrientes como el sodio (169.6\%) y el fósforo (227.3\%) también se encontraron por arriba de los requerimientos diarios. En contraste, el consumo de niacina $(80.6 \%)$ registró un consumo por debajo de la ingesta recomendada.

\section{Terapia Nutricional de Precisión}

\section{Evidencia de investigación en que se basa la terapia:}

1. En un estudio en humanos se compararon 4 dietas durante 12 semanas: (a) alta en ácidos grasos saturados; (b) dos dietas bajas en grasa y altas en hidratos de carbono complejos con ácidos grasos poliinsaturados de cadena larga o placebo. La suplementación consistió en administrar $1.2 \mathrm{~g} / \mathrm{día}$ de ácidos grasos poliinsaturados de cadena larga; los resultados demostraron que los ácidos grasos regulan la activación de SREBP1, controlando la relación de ácidos grasos saturados e insaturado. Para ser más específicos, la transcripción está regulada negativamente por ácidos grasos monoinsaturados y poliinsaturados [13].

2. En otro estudio realizado en personas seropositivas, se suplementó con $4 \mathrm{~g}$ de ácido graso omega $3 ; 465 \mathrm{mg}$ de EPA y $375 \mathrm{mg}$ de DHA, diariamente durante 24 meses, después del tratamiento los pacientes presentaron una disminución de triglicéridos y hubo una tendencia a la disminución de colesterol total [14].

\section{Terapia recomendada}

El paciente de este caso clínico presenta una dislipidemia mixta severa probablemente inducida por el uso de un inhibidor de la proteasa (Lopinavir/Ritonavir), el cuál a pesar de que el paciente tiene un peso normal y que su ingesta calórica es similar a la ingesta energética requerida, no ha logrado disminuir sus niveles de lípidos séricos. En la evaluación dietética se observa un desbalance entre la ingesta de ácidos grasos saturados (SAT) y de los poliinsaturados (PIS), la cual debe corregir. Basado en los antecedentes y condiciones del paciente y retomando la evidencia de los estudios de investigación mencionados, se propone la siguiente terapia nutricional de precisión:

Dieta normo calórica y normo proteica, con la siguiente distribución de macronutrientes: $55 \%$ Hidratos de carbono complejos; $15 \%$ de proteínas y $30 \%$ de lípidos
( $\leq 10$ de SAT, del $6-11 \%$ de PIS $y \leq 9 \%$ de monoinsaturados). Se debe disminuir la ingesta de sodio $(\leq 2.3 \mathrm{~g})$ y de colesterol $(\leq 300 \mathrm{mg})$, incrementando al $100 \%$ del IDR el niacina.

El paciente debe realizar ejercicio aeróbico de moderada intensidad y entrenamiento de resistencia de manera progresiva 5 veces por semana, durante al menos 30 minutos/día. Esto tendrá efectos positivos en la acumulación de grasa corporal, dislipidemias y capacidad aeróbica pero sin cambios en la carga viral y el recuento de CD4+ [15].

Es necesario asegurar la IDR de ácidos grasos poliinsaturados a través de los alimentos de consumo habitual. Para regular la actividad de SREBP, se realizará una suplementación durante 6 meses con $4 \mathrm{~g}$ de ácidos grasos omega 3 (Linolénico, EPA y DHA).

La evaluación del efecto de la terapia será evaluada cada 3 meses, periodo en que se realizaran ajustes al tratamiento en caso necesario.

\section{Referencias}

[1] OMS. Temas de salud: VIH/SIDA 2019. Recuperado de: https://www.who.int/topics/hiv_aids/es/.

[2] Laslett LJ, Alagona P, Clark BA, Drozda JP, Saldivar F, Wilson SR, et al. The worldwide environment of cardiovascular disease: prevalence, diagnosis, therapy, and policy issues: a report from the American College of Cardiology. Journal of the American College of Cardiology. 2012;60(25 Supplement):S1-S49.

[3] Jain RG, Furfine ES, Pedneault L, White AJ, Lenhard JM. Metabolic complications associated with antiretroviral therapy. Antiviral research. 2001;51(3):151-77.

[4] Shamah-Levy T, Cuevas-Nasu L, Rivera-Dommarco J, HernándezÁvila M. Encuesta Nacional de Nutrición y Salud de Medio Camino 2016 (ENSANUT MC 2016). Informe final de resultados. Recuperado de https://www.insp.mx/ensanut/medio-camino-16 html.

[5] ML. G. Evaluation and management of dyslipidemia in patients with HIV infection. JGIM 2002;17:797-810.

[6] Zangerle R SM, Gallati H, Reibnegger G, Wachter H, Fuchs D. Decreased plasma concentrations of HDL cholesterol in HIV-infected individuals are associated with immune activation. J Acquir Immune Defic Syndr 1994;7:1149-56.

[7] Dubé MP, Stein JH, Aberg JA, Fichtenbaum CJ, Gerber JG, Tashima $\mathrm{KT}$, et al. Guidelines for the evaluation and management of dyslipidemia in human immunodeficiency virus (HIV)-infected adults receiving antiretroviral therapy: recommendations of the HIV Medicine Association of the Infectious Disease Society of America and the Adult AIDS Clinical Trials Group. Clinical Infectious Diseases. 2003;37(5):613-27.

[8] Riddle TM, Kuhel DG, Woollett LA, Fichtenbaum CJ, Hui DY. HIV protease inhibitor induces fatty acid and sterol biosynthesis in liver and adipose tissues due to the accumulation of activated sterol regulatory element-binding proteins in the nucleus. Journal of Biological Chemistry. 2001;276(40):37514-9. 
[9] Ferguson LR, De Caterina R, Gorman U, Allayee H, Kohlmeier M, Prasad C, et al. Guide and Position of the International Society of Nutrigenetics/Nutrigenomics on Personalised Nutrition: Part 1 - Fields of Precision Nutrition. Journal of nutrigenetics and nutrigenomics. 2016;9(1):12-27.

[10] Horton JD, Bashmakov Y, Shimomura I, Shimano H. Regulation of sterol regulatory element binding proteins in livers of fasted and refed mice. Proceedings of the National Academy of Sciences. 1998;95(11):5987-92.

[11] Kim JB, Sarraf P, Wright M, Yao KM, Mueller E, Solanes G, et al. Nutritional and insulin regulation of fatty acid synthetase and leptin gene expression through ADD1/SREBP1. The Journal of clinical investigation. 1998;101(1):1-9.

[12] Elmadfa I, Kornsteiner M. Fats and fatty acid requirements for adults. Annals of nutrition \& metabolism. 2009;55(1-3):56-75.

[13] Camargo A, Meneses ME, Pérez-Martínez P, Delgado-Lista J, Rangel-Zúñiga OA, Marín C, et al. Dietary fat modifies lipid metabolism in the adipose tissue of metabolic syndrome patients. Genes \& nutrition. 2014;9(4):409.

[14] Volpe G, Skinner S, Gerrior-Schofield J, Zhang FF, Tang A, Kuvin J, et al., editors. A Randomized Controlled Trial of Omega-3 Fatty Acids in Human Immunodeficiency Virus (HIV): Long-Term Effects on Lipids and Vascular Function. Open Forum Infectious Diseases; 2016: Oxford University Press.

[15] Jaggers JR, Hand GA. Health Benefits of Exercise for People Living With HIV: A Review of the Literature. American journal of lifestyle medicine. 2016;10(3):184-92. 